\title{
3D: The next generation near-infrared imaging spectrometer
}

\author{
L. Weitzel, A. Krabbe, H. Kroker, N. Thatte, L.E. Tacconi-Garman, M. Cameron and R. Genzel \\ Max-Planck Institut für Extraterrestrische Physik (MPE), Postbox 1603, D-85740 Garching, Germany \\ e-mail: krabbe@mpe-garching.mpg.de
}

Received November 9, 1995; accepted February 17, 1996

\begin{abstract}
The new MPE near infrared imaging spectrometer 3D represents a new generation of astronomical instrumentation. It is based on a $256^{2}$ NICMOS-3 Rockwell array and can simultaneously obtain $256 \mathrm{H}$ - or $\mathrm{K}$-band spectra at $R=1100$ or 2100 from a square $16 \times 16$ pixel field on the sky. Typical pixel scales are $0.3^{\prime \prime} /$ pixel or $0.5^{\prime \prime} /$ pixel. $3 \mathrm{D}$ is a combination of a novel image slicer and a liquid nitrogen cooled long slit spectrometer. It includes high definition on-axis lens optics, a high efficiency directly ruled KRS-5 grism as well as a cold closed-loop piezo-driven tilt mirror allowing full spectral sampling. The instrument efficiency including detector is $15 \%$. Combining the advantages of imaging and spectroscopy increases the observing efficiency on key astronomical objects (e.g. galactic nuclei) by such a large factor over existing grating or Fabry-Perot spectrometers that subarcsecond near-IR spectroscopy of faint Seyferts, starbursts, quasars, or distant galaxy clusters becomes feasible for the first time with $4 \mathrm{~m}$-class telescopes. As a portable instrument 3D has already been successfully deployed on several 2 and 4m-class telescopes.
\end{abstract}

Key words: instrumentation: spectrographs; detectors — infrared: general

\section{Introduction}

The technique of imaging spectroscopy in the nearinfrared (NIR, $1.2-2.45 \mu \mathrm{m})$ at subarcsecond scales is only a few years old, but has already proven its potential of making fundamental impacts on various astrophysical issues. "Imaging spectroscopy" or "integral field spectroscopy", as it is sometimes called, means that at every position in a field a spectrum has been obtained and the information content is a 3 dimensional data cube which comprises the spatial information about the observed object in two dimensions and the spectral information in the third dimension.

Two techniques have mainly been applied so far: Stepping the long slit of a (grating) spectrometer across the object and scanning a Fabry-Perot interferometer (FPI) through the wavelength range while staring at the object. Both methods work well at spatial resolutions $\geq 1^{\prime \prime}$ and have shown great results if the objects are bright enough (Krabbe et al. 1991; Burton \& Allen 1992; Krabbe et al. 1993; Lutz et al. 1993; Allen 1994; Gatley \& Merrill 1994; Blietz et al. 1994). However many of the most exciting possible targets tend to be small and relatively faint and if such extended objects (e.g. nuclei of galaxies or high $z$ galaxies) are to be observed on subarcsecond scales both techniques have a few disadvantages:

Send offprint requests to: A. Krabbe
- Both methods can only acquire two of the three dimensions of the data cube at any time. The third dimension has to be obtained sequentially: In the slit spectrometer case it is the second spatial dimension, in the case of the FPI it is the wavelength dimension. This sequential stepping takes a long time and is very inefficient if high spatial resolution and/or good spectral resolution is desired. Assume an object of only $10^{\prime \prime}$ diameter emitting broad lines with $1000 \mathrm{~km} / \mathrm{s}$ FWHM (e.g. an AGN) is to be observed with a spatial sampling of $0.5^{\prime \prime}$ and a spectral resolution of $300 \mathrm{~km} / \mathrm{s}$. In case of the slit spectrometer 20 slit positions, in case of a FPI about 18 wavelength positions per line have to be obtained in order to complete the observation. Observing the $\mathrm{CO}$ bands in the $H$ - or $K$ band presents an even worse case for the FPI. If a single integration requires more than about $1000 \mathrm{~s}$ of integration time, both methods may not be practical any more.

- Both methods add systematic noise to the data. Even bright targets often require a few hours of observing time in order to complete the task. During the integration, atmospheric conditions constantly change and thus influence the quality of the data. The fact that spatially or spectrally related data have to be obtained sequentially now becomes a real handicap; variations of seeing, air mass and $\mathrm{OH}$ emission lines which typically occur during the night as well as imperfect telescope pointing reproducibility will affect different sets of data differently and can make the construction of a homogeneous data cube more difficult 
(Allen et al. 1990), if not impossible. The data from a slit spectrometer are mostly affected by slit position uncertainties and seeing variations, in case of a FPI seeing variations and changes in atmospheric transmission dominate. The combined effect of the contributions mentioned is an increase in the noise level of the spectra and images by a factor of between 3 and 5 . As a solution, one can use shorter integration times per frame and repeat the observing sequence several times. However, the data may then be dominated by detector read-noise rather than background photon noise.

- Both methods do not effectively make use of the field of view (FOV) provided by the instruments. The slit length of NIR long slit spectrometers is typically of the order of $3 \mathrm{arcmin}$. If the object diameter is of the order of $10^{\prime \prime}$, only $11 \%$ of the slit area is effectively used, even if one includes a factor of 2 to account for the fact that sky spectra are required to subtract the background from the on-source measurement. In the case of a FPI the situation is similar: If small objects are being observed a large fraction of the FOV is filled with "blank sky", most of which may be wasted if no other object is in the FOV.

Summarising these arguments, an instrument capable of obtaining a complete data cube in a single integration and at the same time avoiding the excess contributions from atmospheric noise could easily reduce the integration time by a factor of between 10 and 30 compared to conventional instruments, depending on the morphology and the number of interesting NIR spectral lines emitted by the specific object. In addition, such an instrument offers two additional advantages which should not be overlooked:

- It delivers reliable and consistent data under less favourable weather conditions, especially if shorter periods of good weather prevail.

- The requirements for absolute positioning a source are greatly reduced compared to a slit spectrometer. There is no need to exactly place the object within the slit because the FOV is covered with multiple slits. Faint objects have only to be positioned within the FOV which is much easier and often saves effort, especially if the position or morphology is not known exactly. This does not relax the accuracy of the telescope tracking since the object has to remain in the same position on then detector during the integration time.

Such an instrument, called 3D, has been developed at the MPE (Weitzel 1994; Krabbe et al. 1995a) and has been deployed very successfully at a number of 2 and $4 \mathrm{~m}-$ class-telescopes such as the $4.2 \mathrm{~m}$ WHT on La Palma, the $3.5 \mathrm{~m}$ telescope on Calar Alto, and the $2.2 \mathrm{~m} \mathrm{ESO}$ telescope in Chile. In the following sections, the working principle, the optical and mechanical design as well as the electronics of 3D are presented, together with results that have been obtained recently. An introduction to the data reduction procedure has also been added because the data are somewhat more complex in structure in comparison to those delivered by conventional instruments.

\section{Instrument design}

\subsection{The working principle}

The task of imaging a three dimensional data cube onto a two dimensional detector requires a transformation of three dimensions into two. Therefore, the main constituent of $3 \mathrm{D}$ is an image slicer working at ambient temperature, which transforms a two dimensional image into a one dimensional long strip, allowing use of the second dimension of the detector for the spectrum. The long strip exactly matches the entrance slit of a cooled long slit grism spectrometer. The dispersed spectra at the exit of the spectrometer are then imaged onto a $256 \times 256 \mathrm{HgCdTe}$ NICMOS 3 (Rockwell Technologies, Santa Clara, U.S.A.) detector. The instrument, except for the slicer, is cooled to $77 \mathrm{~K}$ using liquid nitrogen to suppress thermal background in the $K$-band $(1.95 \mu \mathrm{m}-2.45 \mu \mathrm{m})$.

The image slicer accepts light within a square field and cuts it into 16 parallel strips which are then rearranged as illustrated in Fig. 1. At this point the image is still multi-chromatic. The fact that the resulting slit is not a straight line but looks stair-like does not affect the performance of the spectrometer, it only shifts the spectra on the detector by a small amount with respect to each other. Currently, the pre-optics in front of the image slicer (ROGUE, Thatte et al. 1995) allows the spatial scale to be set to $0.3^{\prime \prime}$ or $0.5^{\prime \prime} /$ pixel at a $4 \mathrm{~m}$-telescope. The latter represents the highest throughput that the instrument can handle without vignetting.

The optical system of $3 \mathrm{D}$ consists of the following main components: image slicer, collimator, direct ruled grism, camera, detector array, and telescope interface. Figure 5 shows the schematic layout of the optical system of 3D.

\subsection{Image slicer}

Three main types of image slicers have been used in optical astronomical instruments: The Bowen-Walraven slicer (Richardson et al. 1972), the Richardson slicer (Richardson 1972), and optical fibers (Vanderriest \& Lemonnier 1987; Barden \& Wade 1988; Arribas et al. 1991). The Bowen-Walraven slicer uses multiple reflections within the plane parallel surfaces of a quartz plate that is glued to the long side of a specially designed glass prism. The Richardson slicer is a modified White cell which is used in laser technology to extend the optical light path. Both types of slicers are not applicable to 3D. In the Bowen-Walraven slicer, the spatial information is lost after slicing and the Richardson type would be too large and too complicated to adjust.

A slicer using an optical fiber bundle (e.g. SILFID, Vanderriest \& Lemonnier 1987) is a very 


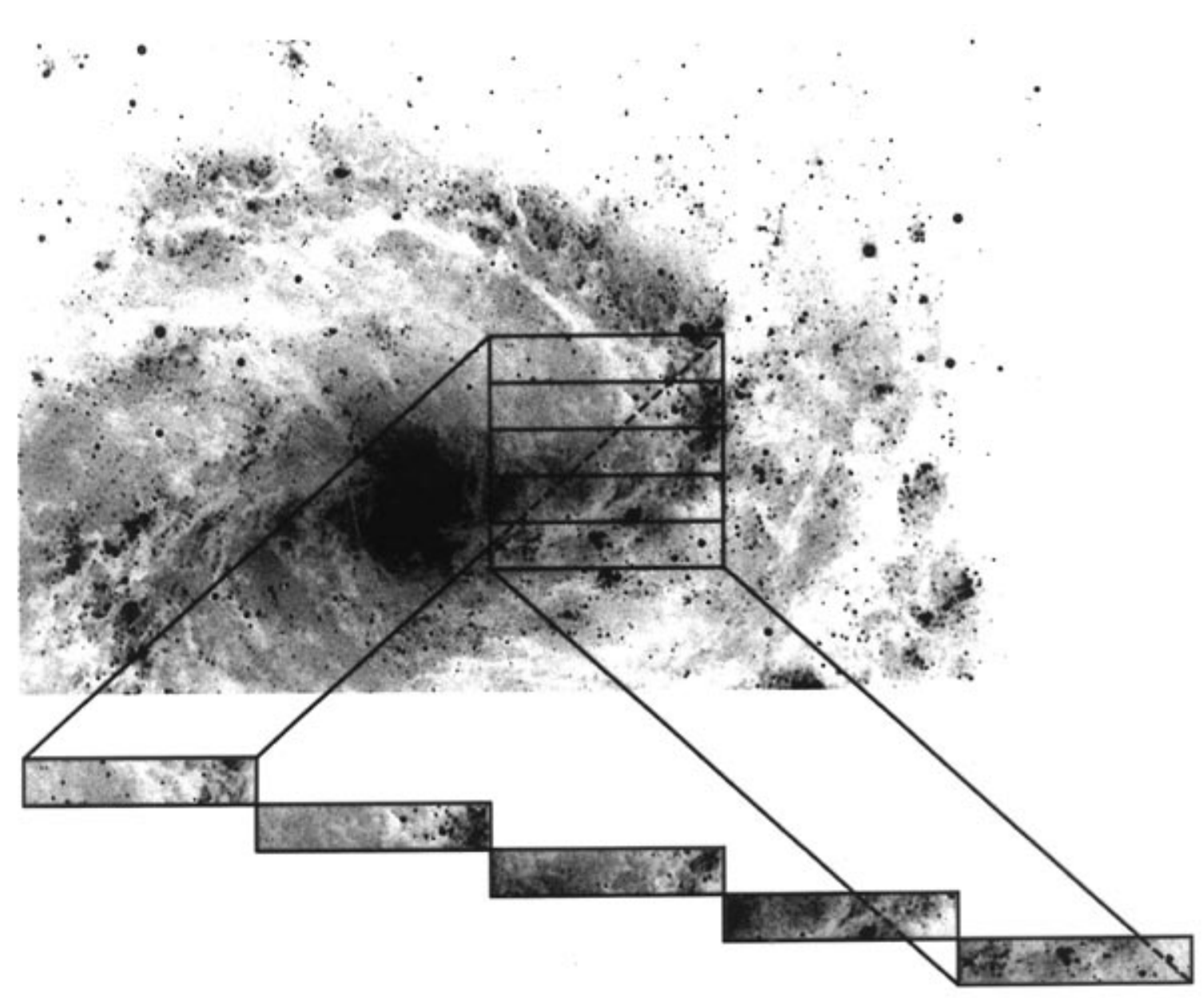

Fig. 1. The working principle of the $3 \mathrm{D}$ image slicer. The upper box represents the field of view with the original image. The image is horizontally cut into 16 strips, demonstrated here with 5 strips only. The single strips are then optically rearranged in the way shown to form a stair-like long slit

attractive solution for visible wavelengths. In the infrared, however, there are a few technical problems associated with fibers. The main disadvantage is the very fast focal ratios of fibers. A typical IR-fiber (e.g. from Le Verre Floure, France) has a numerical aperture ( $n a=$ $\left.\sqrt{n_{\text {core }}^{2}-n_{\text {cladd }}^{2}}\right)$ of about 0.2 , resulting in a focal ratio of 2.5. In order to keep the thermal background as small as possible and to keep the instrument flexible enough to use on different sized telescopes (same pixel scale at different $f$-ratios) such fibers would have to be cold baffled and operated at cryogenic temperatures. At the time of the design review of $3 \mathrm{D}$, four years ago, the properties of fibers at cryogenic temperatures had not been investigated in enough detail to allow their use in a cooled optical system. As such, fiber bundles have only been used at wavelengths up to the $H$-band (e.g. ISIS, Dallier et al. 1994).

A new type of optical slicer was developed for 3D, making imaging spectroscopy in the near-IR feasible for the first time. The 3D image slicer (Fig. 2) is composed of two sets of gold coated multisegment plane mirrors and a mechanical support structure all made of Zerodur. The first set of mirrors slices an image into 16 strips and reflects them into different directions while the second set of mirrors rearranges the individual strips and aligns them into one contiguous long strip such that the virtual pupils of the 16 single strips coincide. The first and smaller stack of mirrors is located at the focal plane of the telescope. Each single mirror is about $8 \mathrm{~mm}$ long and $0.4 \mathrm{~mm}$ wide. The 16 small mirrors and all other parts of the slicer are optically contacted and kept together by molecular adhesive forces only. The second set of mirrors is made as one piece replicated from a master negative. In order to ensure proper functioning of the slicer, the single mirror segments have to be aligned very accurately. The angular accuracy achieved by the 3D slicer is better than $25^{\prime \prime}$ in both directions.

The principal geometry of the slicer type described here and its derivatives is determined by two optical conditions which have to be fulfilled, if the slicer is to work 


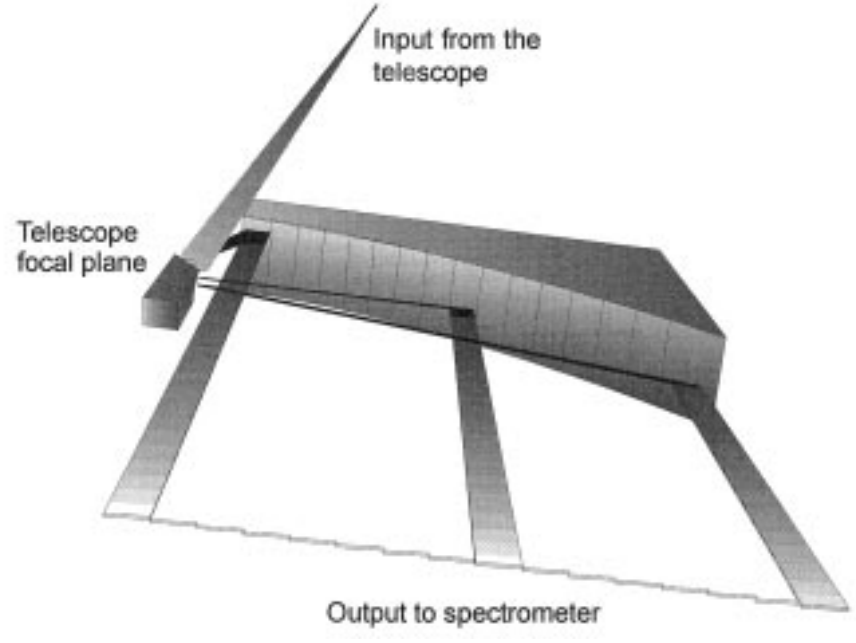

Fig. 2. The 3D image slicer consists of two sets of stacked plane mirrors which in combination rearrange the image to form a stairlike long slit. The small stack in the telescope focal plane cuts the image into 16 horizontal strips, each of which is reflected into a different direction. The second stack is designed such that it realignes the 16 strips in the way shown

correctly. These are the conservation of a common pupil and the equality of the lengths of the 16 optical light paths. Conserving the pupil is important for reducing the background in the optical system, as it minimises the size of the aperture stop. Optically, the reimaged 16 single strips are then almost indistinguishable from a single long slit and can be directly fed into a conventional cryogenic spectrometer. Equal optical path lengths are essential for keeping the 16 pupil positions or 16 focus positions in a common plane.

Both conditions can be matched simultaneously if the area centers of the segments of the second mirror system are aligned along a hyperbola as illustrated in Fig. 3. One focus of the hyperbola coincides with the telescope focus at the location of the first mirror system, while the other focus, which is the new virtual entrance pupil, is placed on the optical axis of the telescope about $2 s$ behind the real telescope pupil (see below, and Fig. 3). In other words, the chief rays passing through the midpoints of the segments of the small mirror system intersect the second system at points located on a hyperbola. The normal vectors of the mirror segments at these intersection points agree with those of the hyperbola (Fig. 3).

The relations between $a, c, p$ and $s$ in a hyperbola are: $c^{2}=a^{2}+b^{2}, p a=b^{2}, s=c-a .2 c$ is the distance between the two foci, $2 a$ is the distance between the vertices of the two hyperbolas. $p$ is the length of $a$ line drawn perpendicular to the optical axis from the focus to the hyperbola. $s$ is the distance between the telescope focus and the vertex of the hyperbola and $b$ is the second hyperbola parameter. Figure 3 illustrates that the distance between telescope pupil and telescope focus is $2 a$, as is the distance between the virtual entrance pupil and the virtual entrance slit. By definition of a hyperbola, the distances $s_{1}$ to $s_{3}$ are then identical with $s_{1}^{\prime}$ to $s_{3}^{\prime}$ proving that the common length of the light paths is conserved.

In this layout there are a few peculiarities which have to be taken into account when designing the optical system of the spectrometer. In fact the virtual images of the 16 slits do not lie on a single plane surface but are aligned along the circumference of a circle as illustrated in Fig. 3 . The entrance slit of the spectrometer is virtual and the direction of the optical axis beyond the slicer slightly differs (by $0.3^{\circ}$ ) from the original telescope axis. The new optical axis is defined by a chief ray passing through the center of the reimaged entrance slit. If a is increased such that the position of the virtual entrance pupil goes to $-\infty$, the reimaged entrance slit lies on a straight line and the hyperbola becomes a parabola. There may be applications where such an optical configuration is desirable, but one has to keep in mind that the distance between the focus of the beams within each strip and their common pupil will remain unchanged with only (arrays of) plane mirrors in the beam.

A second effect is that the images of the slices are tilted at different angles with respect to each other. Both effects are equivalent to a minor defocus of the slices. Therefore, the optical system of the spectrometer has to be designed in such a way that it is able to focus all 16 (slightly defocused) slits onto the array with sufficient image quality. In the present design, the deviation from a single plane surface is very small due to the chosen slicer parameters. These were determined by the following method: The width of the small slicer mirror system determines the size of the slicer as a whole and the size of the optical system of the spectrometer. Therefore, the smallest size technically feasible was chosen, which was $0.4 \mathrm{~mm}$. The other two free parameters in the design of the 3D slicer were the position of the common pupil and the distance between the mirror systems $s$. The position of the pupil was chosen so that the length of the light path from the telescope/common pupil to any point beyond the focus was the same with or without the slicer (conserving the focus-pupil distance). The other parameter, the distance between the two slicer parts, was determined by optimising the light efficiency of the slicer using geometrical optics only.

The light efficiency is not $100 \%$, since the small slicer mirror segments are tilted with respect to the focal plane. Therefore none of their surfaces are in focus, resulting in a loss of light at the edges of the segments due to beam divergence. Furthermore, the segments on the second set of mirrors cut off radiation from the defocused incoming beams at their edges. This is due to the fact that the sizes of the mirror segments cannot be chosen at will, but are constrained by their neighbours so as to avoid overlap. The total efficiency of the slicer (all 16 slits) is 94\% using 


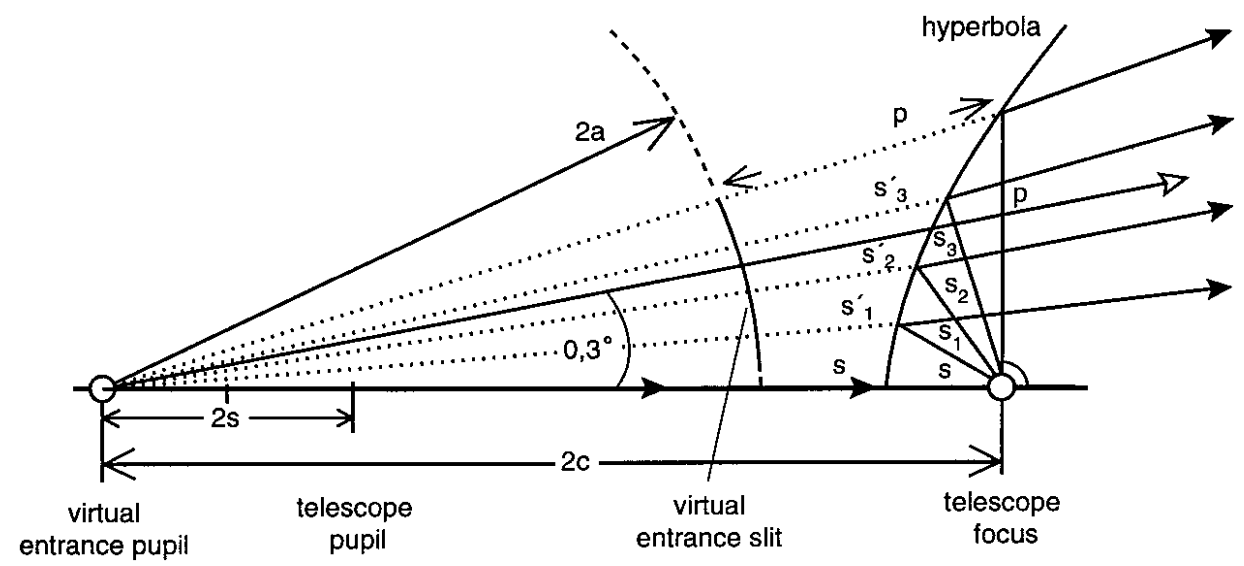

Fig. 3. Mathematical relations determining the geometry of the $3 \mathrm{D}$ image slicer: The chief rays from the individual slices originate from a single virtual pupil on the optical axis of telescope. For details see text

geometrical optics only. Light loss due to Fresnel diffraction at the small slicer mirrors is minimal $(<3 \%)$.

Figure 4 shows other possible optical configurations of this slicer principle. The symmetric version (Fig. 4a) allows for an even more compact design, but has the disadvantage that the central slit position cannot be used. It collides with the incoming beam and the reflected light is blocked by the small segmented mirror. A possible solution at the cost of higher complexity is a slight tilt of the image slicer with respect to the optical axis. This allows the incoming beam to pass underneath the big segment mirror and the outgoing beam to pass over the small segment mirror (Fig. 4b).

\subsection{Collimator-camera system}

The 3D collimator-camera system consists entirely of lenses with spherical surfaces complemented by three flat folding mirrors. Lenses provide easy optical alignment and are not as sensitive to structural bending of the mounting plate as mirrors, thus simplifying the mechanical design for a cryogenic instrument. The optical system was designed using the Kidger Optics SIGMA 5.7/5.9 (Kidger Optics, Crowborough, UK) software for operation at $77 \mathrm{~K}$ and so as to match the $3.5 \mathrm{~m}$ Calar Alto telescope in the IR-f/45 configuration. The optical design was mainly constrained by the fact that the $100 \mathrm{~mm}$ long entrance slit had to be imaged onto the detector such that its length exactly matched the 256 pixel rows of the detector. This requires the slit width to exactly match the detector pixel-to-pixel spacing of $40 \mu \mathrm{m}$, thus demanding a demagnification of the entrance slit by a factor of 10 . The absolute focal length of the collimator is a compromise between the desire for a compact instrument and the demand that curvature of the exit slit should be small enough not to degrade the spectral resolution and to be easily corrected by the data reduction software.
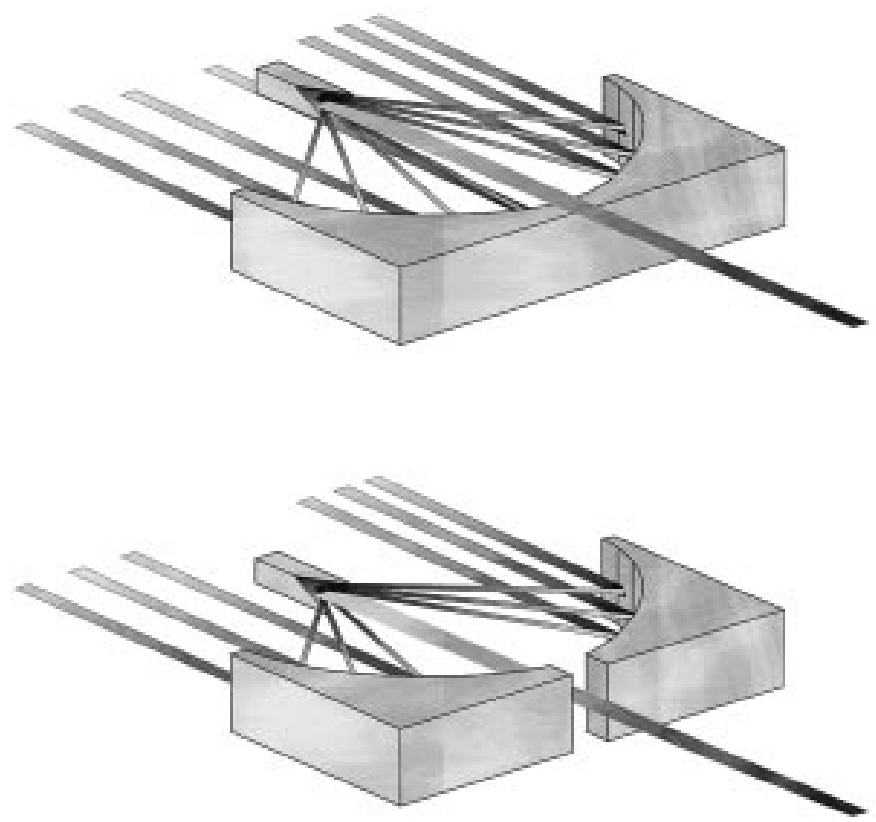

Fig. 4. Other possible configurations of the 3D slicer principle: The symmetric optical set-up (bottom) avoids large deflection angles but shades off the central slit position. This can be avoided at the cost of higher optical complexity by rotating the image slicer with respect to the focal plane of the telescope by a small angle such that the mirror structure avoids intersecting with the telescope beam as well as with the small mirror stack (top)

The result is shown in Fig. 5 and summarised in Table 1 . The collimator and camera are each designed as 3 -lens systems. The collimator lenses are cut to their minimum required size in order to save weight and space, to increase their stability against temperature changes, and to reduce the cool down time of the whole instrument. The image quality provided by the optical system is 
excellent in the $K$-band and adequate in the $H$-band. For $K$-band spectroscopy the geometrical blur at every position in the camera focal plane is smaller than $10 \mu \mathrm{m}$ as Fig. 6 demonstrates. All the lenses are coated with a broadband anti-reflection coating covering the $H$ - and $K$-bands. The optical transmission of $3 \mathrm{D}$ from the entrance window to the last camera lens including the $K$-band grism and the $K$-band filter is about $35 \%$.

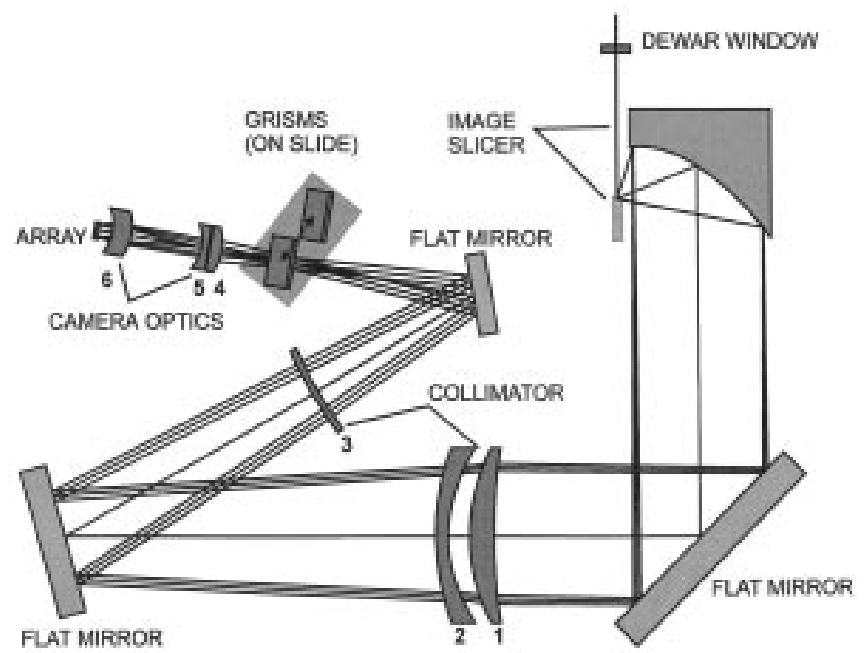

Fig. 5. Optical layout of 3D. The instrument is cooled to 77 $\mathrm{K}$ except for the slicer and the next flat mirror upstream. The flat mirrors minimize the required space

The three flat mirrors are made from Zerodur. The second flat mirror can be tilted vertically thus slightly shifting the spectrum across the detector in order to provide fully sampled spectra (Kroker 1996b).

\subsection{Grism}

The decision in favour of grisms instead of a reflection grating was mainly based on three arguments: The use of grisms allows for a much more compact design, as the lenses can be placed close to the dispersing element; a grism introduces less optical aberrations (Schroeder 1987); the optical adjustment is simpler with the consequence that the exchange of grisms is straightforward. A grism is usually designed such that the light enters through the flat front surface perpendicular to the beam and exits through the grating area. For a selectable (blaze) wavelength the beam passes straight through without any deflection. As a result, a grism is preferentially used on-axis allowing a compact optical design.

Grisms have a few disadvantages as well. Their nonlinear dispersion is due to the fact that a grism is a combination of prism and grating and the optical path differences at the grooves are directly related to the dispersion of the prism material. The dispersion of a grism is smaller by a factor of $2 /(n-1)$ compared with a reflection grating with the same parameters where $n$ is the refractive index. A high refractive index is therefore desirable because it keeps the prism angle and, consequently, the mechanical size of the grism small and yields a higher grating efficiency averaged over the free spectral range. The critical issue with grisms is the choice of material. The 3D grisms are made out of KRS- 5 with a refractive index of 2.4 in the near IR. When using high refractive index materials, direct ruled grisms are the only feasible alternative: A replicated grism would suffer from total internal reflection at the prism-grating transition due to the low refractive index of the resin $\left(n_{\mathrm{NIR}} \sim 1.56\right)$. If on the other hand, the prism angle of a low refractive index material had to be larger than $40^{\circ}$, the blaze wavelength is shifted with respect to the value calculated from geometrical optics: $\lambda_{\text {blaze }}=\sigma / m(n-1) \sin (\alpha)$, where $\sigma$ is the grating constant and $\alpha$ is the smaller prism angle. The shift occurs because simple scalar grism theory is not applicable at these angles and the full electromagnetic theory is required instead (Neviere 1991; Dekker et al. 1988). An instrument design which allows for the conservation of the optical axis would consequently require a substantial amount of optimisation to find the right grism parameters. For the two high resolution $K$-band grisms in 3D possible shifts in $\lambda_{\text {blaze }}$ do not yet have to be taken into account as has been checked numerically by Neviere (1994).

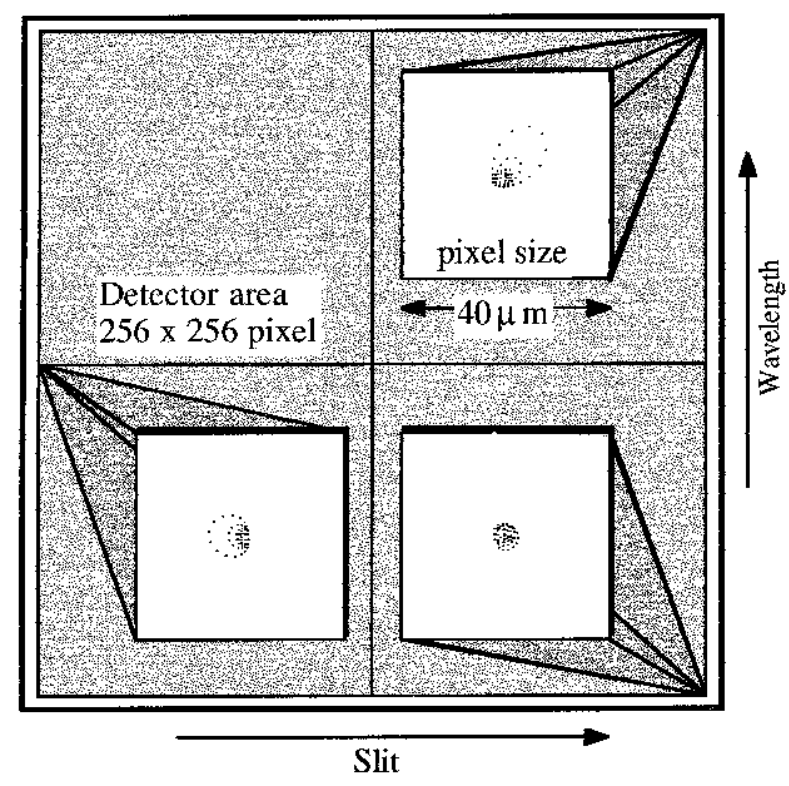

Fig. 6. Spot diagram of the of $3 \mathrm{D}$ optics using a $R=1000$ $K$-band grism in the upper and lower right corners as well as on the middle of the left side. The diameter of the spots is much smaller compared with the $40 \mu \mathrm{m} \times 40 \mu \mathrm{m}$ pixel size of the detector and demonstrates the excellent optical quality of the $3 \mathrm{D}$ optics

The difficulty in manufacturing direct ruled grisms is the lack of high refractive index materials possessing the 
Table 1. Parameter of the $3 \mathrm{D}$ optical system at $77 \mathrm{~K}$. All measures in $[\mathrm{mm}]$

\begin{tabular}{|c|c|c|c|c|c|c|}
\hline \multirow{2}{*}{ Lens } & \multirow[t]{2}{*}{ Material } & \multicolumn{2}{|c|}{ Radius } & \multirow[t]{2}{*}{ Thickness } & \multirow{2}{*}{$\begin{array}{l}\text { Distance to pre- } \\
\text { vious element }\end{array}$} & \multirow[t]{2}{*}{$\varnothing$} \\
\hline & & front & back & & & \\
\hline 1 & $\mathrm{BaF}_{2}$ & -1087.872 & 140.053 & 20.395 & 499.368 & 130 \\
\hline 2 & $\mathrm{CaF}_{2}$ & -129.744 & 293.391 & 5.518 & 14.301 & 130 \\
\hline 3 & $\mathrm{CaF}_{2}$ & 244.004 & -363.858 & 4.987 & 505.761 & 70 \\
\hline 4 & ZnS & 47.457 & -474.003 & 5.600 & 336.294 & 36 \\
\hline 5 & $\mathrm{CaF}_{2}$ & -135.512 & -27.985 & 3.457 & 3.632 & 36 \\
\hline 6 & $\mathrm{ZnS}$ & 35.907 & -42.682 & 15.036 & 57.626 & 36 \\
\hline
\end{tabular}

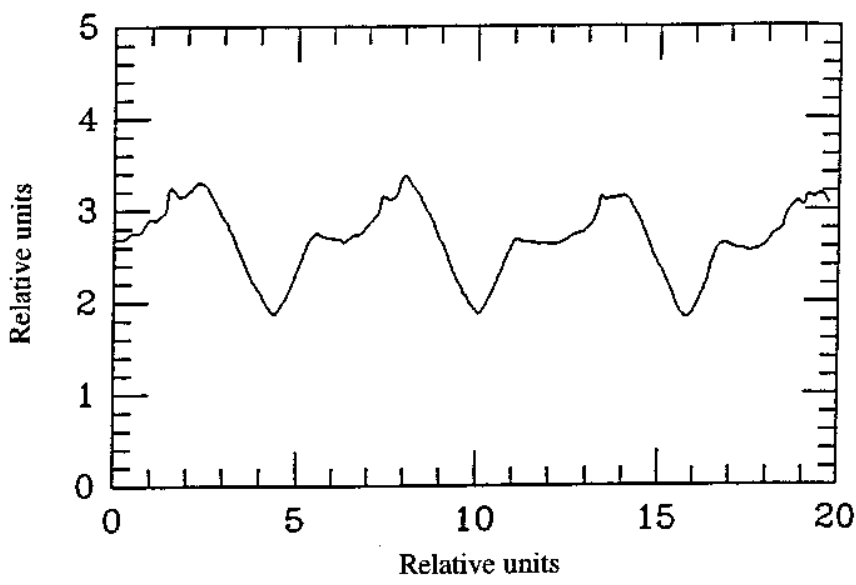

Fig. 7. a) Measured groove profile of a CdTe test grism (Zeiss, Jena, Germany). The broken edges of the grooves create a high straylight level and thus low efficiency

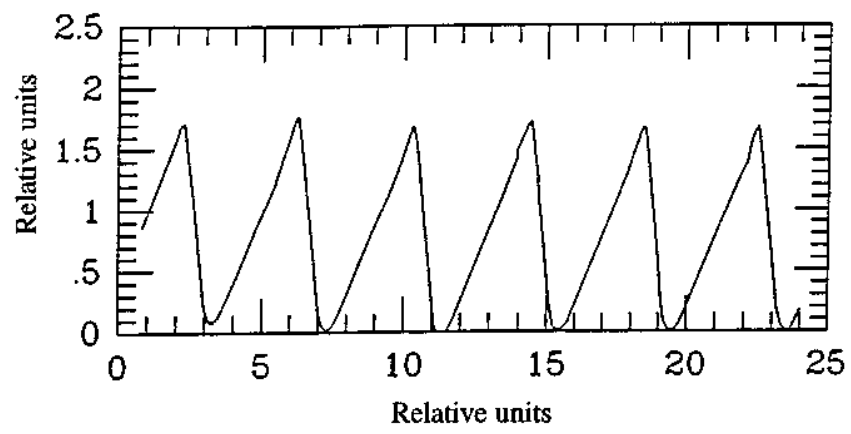

Fig. 7. b) Measured groove profile of the $3 \mathrm{D} K$-band $R=1000$ KRS-5 grism (Zeiss, Jena, Germany). The grating angle is $24^{\circ}$ and the grating constant is $7.68 \mu \mathrm{m}$ (Weitzel 1994)

right mechanical properties which are necessary to obtain good groove profiles leading to high grating efficiencies. For 3D, KRS-5, a compound of TlBr and TlI with excellent transmission and low hardness, was chosen as the grating material. The relative softness of KRS- 5 compared to other crystals like ZnSe together with its availability as single crystals turned out to be the key elements in achieving excellent groove profiles and consequently little straylight. Tests with other materials failed completely or did not show a sufficient groove profile quality. As an example, Fig. 7a shows the results from mechanical ruling tests using CdTe (Zeiss, Jena, Germany). The 3D grisms (Zeiss, Jena, Germany), by comparison, show a much better groove profile (Fig. $7 \mathrm{~b}$ ) yielding a total grating efficiency of $\sim 60 \%$ which put them amongst the most efficient direct ruled near-IR grisms that exist.

Currently four grisms are available for 3D covering the $H$ - and $K$ - bands at $R=1000$ and the $K$-band at $R=2100$. Details are given in Table 2. The entrance area is antireflection coated resulting in a front surface transmission higher than $98 \%$ over the entire wavelength range.

\subsection{Detector electronics and data acquisition}

The 3D detector has a pixel size of $40 \mu \mathrm{m} \times 40 \mu \mathrm{m}$. In one dimension, a pixel constrains the spectral resolution (e.g. to $R=1100$ with the $K$-band grism); in the other dimension it defines the pixel size in Right Ascension (The pixel size in Declination is defined by the slicer.) The detector is electronically divided into four quadrants, each of which is separately controlled by standard Infrared Laboratories INC., (Tucson, U.S.A.) readout electronics, in a manner similar to the MPE speckle camera SHARP (Hofmann et al. 1992). Among the various read out schemes available, only the correlated double sampling has been used for the observations because it gives the lowest effective read noise. In this clocking scheme, a RESET command followed by two non-destructive READ commands is used. The time interval between the two READs is the integration time which can be selected. Thus, two images are read out during a complete sequence. The data from the four detector quadrants are sent via a fiber link to 4 independent digital signal processors (DSPs) (Fig. 8). The DSPs subtract the pre-exposure image from the post-exposure image for each quadrant. The control VME computer then receives the images, mosaics them together and stores the result on disk. The VME computer is also used to control other parts of the instrument, such as the shutter and the piezo driven mirror. 
Table 2. Parameters of the KRS-5 grisms available for 3D

\begin{tabular}{|l|c|c|c|c|c|c|c|c|c||}
\hline Grism & $\theta_{\text {blaze }}$ & $\lambda_{\text {blaze }}$ & Range $[\mu \mathrm{m}]$ & $\mathrm{m}$ & $\begin{array}{c}\text { Lines } \\
/ \mathrm{mm}\end{array}$ & $\lambda / \Delta \lambda$ & $\begin{array}{c}\text { Ruled area } \\
{\left[\mathrm{mm}^{2}\right]}\end{array}$ & efficiency & $\begin{array}{c}\text { Reflection on } \\
\text { prism front }\end{array}$ \\
\hline $\mathrm{H}$ & $27^{\circ}$ & 1.63 & $1.48-1.78$ & 3 & 130.2 & 1250 & $35 \times 32$ & $60 \%$ & $<1 \%$ \\
$\mathrm{~K}$ & $24^{\circ}$ & 2.17 & $1.94-2.41$ & 2 & 130.2 & 1100 & $35 \times 32$ & $60 \%$ & $<2 \%$ \\
$\mathrm{~K}_{\text {short }}$ & $40^{\circ}$ & 2.06 & $1.95-2.18$ & 2 & 217 & 2100 & $37 \times 32$ & $55 \%$ & $<1 \%$ \\
$\mathrm{~K}_{\text {long }}$ & $40^{\circ}$ & 2.29 & $2.17-2.43$ & 2 & 195 & 2100 & $37 \times 32$ & $55 \%$ & $<1 \%$ \\
\hline
\end{tabular}

The combination DSP/VME computer also provides a first step on-line reduction of the data e.g. a wavelength collapsed restored broad band image. This is of particular importance for an instrument like 3D where one data frame represents a three dimensional data cube and simply viewing a background subtracted image does not reveal enough information to the untrained user to make on-line decisions such as determining telescope pointing offsets.

A careful consideration of the grounding concept in both the analog and digital branches of the IRLaboratories electronics led to several modifications which reduced the single read noise of the detector by a factor of two to $25 \mathrm{e}^{-}$. This increased the instrument sensitivity in the parts of the $H$ - and $K$-bands between the $\mathrm{OH}$ lines, which are generally detector noise dominated, by a corresponding factor. A multiple read readout scheme (Fowler \& Gatley 1990), which should reduce the read noise even further, is currently being implemented.

\subsection{Telescope interface}

$3 \mathrm{D}$ was designed to be directly attached to the $f / 45$-IRCassegrain focus at the $3.5 \mathrm{~m}$ telescope on Calar Alto, Spain. Additional optical interfaces were designed in order to operate the instrument at other telescopes such as the $2.2 \mathrm{~m}$ telescope $(f / 35)$ on La Silla, Chile or the 4.2 m-WHT $(f / 11)$ on La Palma, Spain. The WHT represents the limit in adaptability since the dimensions of the internal optics limit the coupling to the input beam at $0.5^{\prime \prime} /$ pixel spatial scale. On the other hand, if the input beam is much slower than $f / 100$ the spectral resolution will drop in accordance with the reduction in size of the illuminated grism area. All optical components in the interfaces are spherical lenses designed with the Kidger Optics SIGMA 5.7/5.9 software.

The telescope interface also comprises the calibration facilities which can be introduced into the light path by a motor driven slide. These include a black body and Ne, $\mathrm{Kr}$, and Ar discharge lamps. The black body is a temperature calibrated filament lamp with a quartz window (OSRAM, Germany) operated at $T \sim 1300 \mathrm{~K} \& 1750 \mathrm{~K}$. Its spectrum approximates that of a blackbody and provides a featureless, almost flat spectrum in the $K$ - and $H$-bands respectively. Alternatively, a Nernst-glower rod can also be used.

\section{7. $3 D$ cryostat}

A liquid nitrogen $\left(\mathrm{LN}_{2}\right)$ cooled cryostat based on the MPE FIFI instrument (Geis 1990) was designed for 3D. The optical system of the spectrometer is assembled on a $45 \mathrm{~cm}$ $\times 45 \mathrm{~cm}$ cold plate in the cryostat. The image slicer is mounted in the warm part of the cryostat surrounded by cold baffles. The $\mathrm{LN}_{2}$ tank capacity is $20 \mathrm{l}$ providing a hold time $\geq 24$ hours allowing more than one night's worth of observation without refilling. All parts of the cryostat, including the holders for the optical elements are made from tempered aluminium (AlMg4.5Mn), which results not only in a low weight $(\sim 80 \mathrm{~kg})$ instrument but in addition avoids internal thermal stresses between the different parts due to different thermal expansion coefficients. The lens and mirror holders are based on those self centering spring-type mounts developed for FAST (Krabbe et al. 1993). Special care was taken mounting the grisms as KRS- 5 tends to turn opaque and milky under external pressure. No degradation in the performance of the grisms could be measured even after more than 20 cooling cycles.

The few moving components within the cryostat are the grism slide, the piezo-driven tilting mirror and the shutter. The grism slide sits at the position of the pupil and can hold two grisms at a time. It has a mechanical connection to the outside of the cryostat via a ferrofluidic feedthrough. The axis is motor driven and allows for the exchange of the two grisms on the fly (Kroker 1996b). The tilting mirror providing full spectral sampling is driven by means of a piezo translation stage modified for cryogenic temperatures (Queensgate Instruments, UK). Since the response of the piezo ceramic is nonlinear, due to hysteresis and thermal and electrical drifts, a closed loop position control using a capacitive sensor as feedback was added (Queensgate Instruments), which allowed us to achieve a long-term positioning stability of better than $1 / 15$ pixel measured on the detector. The cold shutter blade sits in front of the camera and is driven by a stepper motor which was specifically modified for cryogenic temperature application. It only needs an electrical connection to the outside as does the piezo unit for the moving mirror. The small number of moving parts provides 3D with an excellent reliability which is essential for a travelling instrument.

The Rapid Off-Axis GUider Experiment (ROGUE) is a high sensitivity first order seeing corrector $\left(V_{\max } \sim\right.$ 
3D DATA ACQUISITION SYSTEM

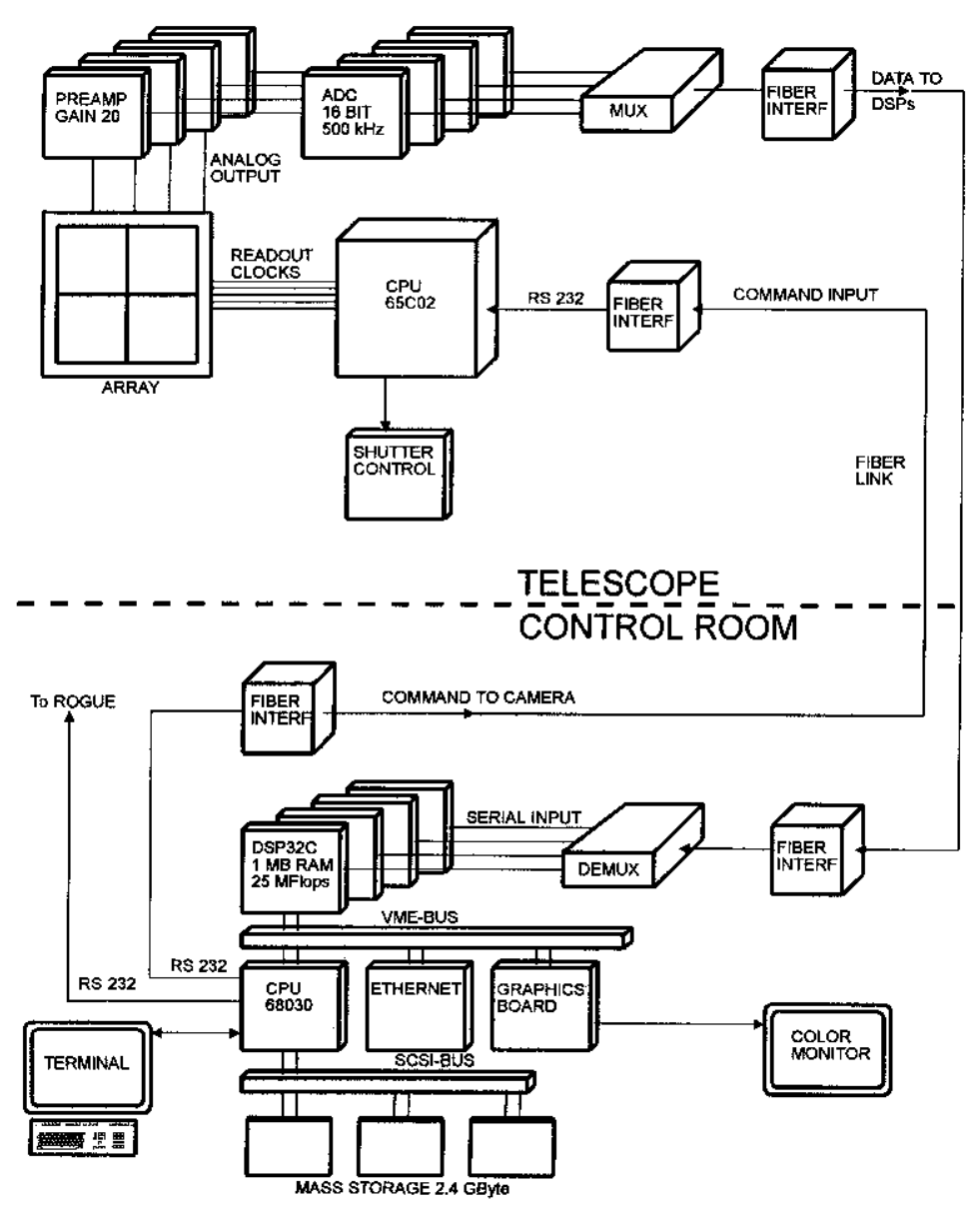

Fig. 8. Block diagramm of the $3 \mathrm{D}$ electronics

$18^{\text {mag }}$ at 4 m-telescope) which uses a piezo controlled mirror located at the pupil plane in order to correct for image motion in real time with a correction frequency of $70 \mathrm{~Hz}$. The isokinetic patch is well covered by its field of view of 4 arcmin. It has been developed to improve the spatial resolution of $3 \mathrm{D}$ but can be applied to other NIR instruments as well. A full description is given by Thatte et al. (1995).

\section{Data reduction}

The 3D data reduction is based on the GIPSY software package (van der Hulst et al. 1992) because it handles data cubes very effectively and provides a user friendly interface to implement dedicated additional software written by the user. The data reduction procedure described below includes standard routines provided by GIPSY as well as about 20 in-house 3D-specific programs.

\subsection{Raw data}

A typical $H$-band raw data frame is shown in Fig. 9a. It more or less resembles the output from a long slit spec- trometer. The vertical axis represents wavelength while the horizontal axis represents both spatial dimensions along the slit. It consists of 16 adjacent slits which are slightly shifted in wavelength with respect to each other. This reflects the fact that the individual slits are not fully aligned at the entrance of the spectrometer but only touch each other at one corner (see Fig. 1). Figure 9a is dominated by atmospheric $\mathrm{OH}$ emission lines and some additional amount of straylight. These contaminations together with the dark current of the detector are removed by subtracting a blank sky image taken in the vicinity the object with the same integration time. The result of the subtraction - already wavelength calibrated - is shown in Fig. 9b, which reveals the existence of continuum emission from an object (a galaxy) in the data as well as several fainter emission and absorption lines. Information about the morphology of the object cannot, however, be easily discerned at this stage of the data reduction. 
Fig. 9. a) Raw $3 \mathrm{D} H$-band data frame. The 16 single slits are clearly visible. The vertical structure is continuum emission from an extended object, the white spot is a cluster of bad pixels. The horizontal stairlike emission are telluric $\mathrm{OH}$ emission lines reflecting the shape of the input slit (see Figs. 1 and 2)

Fig. 9. b) The sky subtracted and wavelength calibrated 3D $H$-band data frame from a) has a full size of $256 \times 300$ pixel. Shown here is a $256 \times 256$ subframe which essentially contains all the useful information. The object is an extended galaxy showing faint emission and absorption lines and some residuals telluric $\mathrm{OH}$ lines

\subsection{Flatfielding and calibration}

Before the two dimensional image can be converted into a three dimensional data cube, it must be flatfielded and wavelength calibrated.

The unique nature of 3D implies that the source used for flat fielding must be both spatially and spectrally flat. As no source satisfies both these constraints simultaneously, the flat field is created from two separate images, one contributing the spatial flat field and the other contributing the spectral flat field. Usually the spatial flat field is obtained from a differential image of the dome (light on/off). Collapsing each of the spectra within the same wavelength interval results in one line of pixels representing the broad-band flux of the dome. As the spectral characteristics are the same in all 256 spectra, the pixel to pixel variations along the line should only be attributable to irregularities in the instrument transmission and the detector response.

The spectral flat field is obtained from the filamentlamp or the Nernst-glower in a similar fashion. If the temperature is properly selected, the spectrum is almost flat within the observed wavelength range. If a differential image (lamp/glower on/off) is normalised to the same intensity over a given wavelength range in each spectrum, differences between the 256 spectra are then attributable only to irregularities in the detector response. The overall flat field is thus created by multiplying every row of the normalised spectral flatfield with the collapsed spatial flatfield and normalising the final image.

It should be noted that an accurate knowledge of the spectral shape of the filament-lamp or Nernst-glower is not critical to the data reduction process. In most cases, the observed spectrum of the source under study is divided by the spectrum of a template star, in order to remove any spectral features introduced by the atmosphere. If both sets of data, the template star as well as the object, have been processed with the same flat field, the unknown calibration spectrum will not affect the data.

Each of the 256 spectra has to be wavelength calibrated in two steps: The wavelength of each pixel has to be determined and the spectra have to be resampled on a regular wavelength grid common to all spectra. The first step is performed by comparing the measured emission lines of a spectral calibration source (neon lamp for $K$-band, Krypton/Argon lamps or telluric $\mathrm{OH}$ lines for the $\mathrm{H}$ band) with the theoretical spectrum. The spectral redistribution is then achieved by interpolating the data in each column of the original image by means of polynomial interpolation. The grid spacing is $2 \mathrm{~nm}$ for the $K$ band and $1.2 \mathrm{~nm}$ for the $H$-band, the mean values in all 256 spectra. The fact that the original spectra are shifted relative to each other has the effect that the wavelength calibrated images contains more than 256 pixels in wavelength dimension (The actual size of 300 was selected as a convenient number). In the calibrated image, extended 
emission and absorption lines form horizontal lines across (part of) the image. Therefore, row number (increasing with increasing $\lambda$ ) can be converted into wavelength. In a calibrated $K$-band data set the transformation is: $\lambda[\mu \mathrm{m}]=$ row $\# * 0.002 "+1.85$, whereas $\lambda[\mu \mathrm{m}]=$ row $\# *^{*} 0.0012+1.45$ holds for the $H$-band $(1 \leq$ row $\# \leq 300)$. If fully sampled spectra are obtained they should be merged before the second step of wavelength calibration takes place. For merged spectra the grid spacing is $1 \mathrm{~nm}$ for the $K$-band and 0.6 $\mathrm{nm}$ for the $H$-band and the total depth of the data cube grows to 600 pixels.

\subsection{Data cube and bad pixel correction}

Each image slice is designed to span exactly 16 detector pixels, 16 such slices constituting the complete sky image. Practical limitations in fabrication of the optical elements as well as imperfect knowledge of refractive indices at 77 $\mathrm{K}$ lead in reality to a total image extent of 252.8 pixels, instead of the desired 256 pixels. Small errors in the optical adjustment between the individual image slicer mirrors can also introduce variations in the number of detector pixels spanned by each image slice. In order to reconstruct the two dimensional image from the 16 image slices, it is necessary to determine the relative shift between the center points of the slices. An exposure is made while moving a reference pinhole across the entire FOV in a north-south direction. The image slices are then aligned so as to yield an image smeared precisely in a vertical direction, allowing us to determine the relative shift of each slice to a fraction of a pixel. The shift values are not integers, making it necessary to re-sample the data from each spatial slice. The re-sampling is carried out either by interpolating or using FFT techniques, since unlike the spectral dimension, the spatial data is adequately sampled in most cases. The edges of each slice often contain pixels receiving light from two spatial slices. These pixels do not contain any useful information in most cases, and can be discarded.

Each data cube is accompanied by a mask cube of the same size and format as the data cube. Every pixel in the mask cube contains information about the validity and integration time of the corresponding pixel in the data cube. This information is necessary if, for example, data cubes with different integration times should be merged or bad pixels interpolated (see below).

Permanent bad pixels are included in a bad pixel list which is copied to the mask cube. Transient unusable pixels due to cosmic ray hits or overexposure are detected by an algorithm and marked in the mask cube as well. If necessary, the bad pixels can be interpolated using a 3 dimensional interpolation routine. The routine includes criteria to determine conditions for a successful interpolation and interpolates bad pixels which match these criteria.

\subsection{Recentering and merging data cubes}

A set of observations usually includes several short integrations on the desired object which need to be reduced separately. Typical reasons are that either the telescope was moved between integrations or the individual frames contain cosmic ray hits which have to be eliminated separately or images have been taken with different integration times. During the standard reduction procedure, 3D data cubes can be spatially shifted on a finer grid such that a recognisable feature in the collapsed broad band image is spatially centered. The procedure can be automated for the brightest pixel, usually the continuum peak in the broad band images. The masks are shifted appropriately as well. Single cubes are merged into the final data cube by averaging them. The final data cube is normalised to one second on-source integration time.

\subsection{Telluric absorption}

Telluric absorption lines can be corrected by ratioing the data cube with a pure atmospheric spectrum. It is, however, impossible to obtain such an ideal spectrum since there is no spectrally smooth background source available. The atmosphere can, however, be approximated by either a model atmosphere or a measured spectrum of a sufficiently bright star obtained at the same air mass as the object.

The model atmosphere from the ATRAN software package (Lord 1992) is currently used to correct $H$-band data and gives satisfactory results. In this band, the telluric absorptions are not very deep and stars are inappropriate because all types show strong spectral emission or absorption lines. In the $K$-band, the situation is the other way around: The deep telluric absorption lines vary strongly with zenith-angle and cannot be sufficiently well reproduced by the ATRAN program. However, stars of spectral types F6III-V to G2III-V show only weak spectral features in the $K$-band which makes them good candidates to serve as atmospheric calibrators. Slight differences in air mass between the object and the calibration star often result in residual telluric lines around $2.0 \mu \mathrm{m}$. These residuals are reduced by transforming the stellar spectrum to the air mass of the object by multiplying the stellar spectrum with the ratio of two model ATRAN $K$-band spectra computed for the air masses of the object and the calibration star. To completely eliminate spectral features introduced by the calibration star, the stellar spectrum is divided by a high-quality transmission corrected reference spectrum obtained from the literature (e.g. Kleinmann \& Hall 1986). The only caveat is that a good reference spectrum for the specific spectral type must be available in the literature. 


\subsection{Absolute calibration and sensitivity}

The data cube is finally flux calibrated by calculating the counts-to-flux conversion factor in units of $\left[\mathrm{W} \mathrm{m}^{-2}\right.$ pixel $^{-1}$ $\mathrm{mm}^{-1}$ counts $^{-1}$ ] from the measurements of a flux standard star. This star is analysed using the reduction software in exactly the same way as the object ensuring that both data sets are directly comparable.

The sensitivity of 3D has been derived from several faint objects observed at the $3.5 \mathrm{~m}$ telescope on Calar Alto, among them IRAS F10214+4724 (see below). Table 3 lists the on-source integration times for a continuum source with a given $K$-band magnitude, in 1 arcsecond seeing at the WHT, for a SNR of 3. The numbers refer to 1 channel, at a nominal wavelength of $2.15 \mu \mathrm{m}$, with integration times for a single frame which exceed 100 seconds at $0.5^{\prime \prime} /$ pixel or 250 seconds at $0.3^{\prime \prime} /$ pixel spatial scale. Note that an equal amount of time has to be spent offsource, in order to calibrate the variable sky background. For line fluxes in extended objects, Table 4 lists the onsource integration times required to achieve a SNR of 3 at $2.15 \mu \mathrm{m}$ in 1 channel at the WHT. The integration times for individual frames are the same as in the previous table.

Table 3.

\begin{tabular}{|c|c|c|c|}
\hline $\begin{array}{l}\text { Point source } \\
\text { K magnitude }\end{array}$ & $0.5^{\prime \prime}$ seeing & $1^{\prime \prime}$ seeing & $1.5^{\prime \prime}$ seeing \\
\hline 15 & $<60 \mathrm{sec}$ & $75 \mathrm{sec}$ & $170 \mathrm{sec}$ \\
\hline 17 & $750 \mathrm{sec}$ & $3000 \mathrm{sec}$ & $6800 \mathrm{sec}$ \\
\hline
\end{tabular}

Table 4.

\begin{tabular}{|c|c|c||}
\hline Flux $\left[\mathrm{W} \mathrm{m}^{-2} \mathrm{q}^{\prime-2}\right]$ & $0.5^{\prime \prime} /$ pixel & $0.3^{\prime \prime} / \mathrm{pixel}$ \\
\hline $1 \times 10^{-19}$ & $17400 \mathrm{sec}$ & - \\
$1 \times 10^{-18}$ & $175 \mathrm{sec}$ & $480 \mathrm{sec}$ \\
\hline
\end{tabular}

\section{Astronomical results}

A number of exciting open questions of infrared astronomy deal with the exploration of subarcsecond structures in both galactic and extragalactic sources. Such issues have motivated us in building $3 \mathrm{D}$ and in this section we present a selection from recent results which, we feel, are representative of what kind of science can be pursued with a 3D-type instrument.

\subsection{The central stellar cluster of our galaxy}

There is an ongoing debate about the nature of the central source in our Galaxy and the source of luminosity in the central parsec (Genzel et al. 1994 and references therein). $3 \mathrm{D}$ observations of a 20 arcsecond radius region, centered on the nucleus of our Galaxy, made at the $2.2 \mathrm{~m}$ ESO telescope, have, for the first time, delivered complete $K$-band spectra $(\lambda / \Delta \lambda=1000)$ of more than 200 stars. An extract, shown in Fig. 10, displays a speckle $K$-band image of the galactic center (Eckart et al. 1995) convolved to 0.4" spatial resolution together with 12 stellar spectra of several stellar types obtained with $3 \mathrm{D}$ in the central $10 \times 10$ arcsec field around $\mathrm{SgrA}^{*}$ (Krabbe et al. 1995b). Late type giants and supergiants are identified by their $\mathrm{CO}$ overtone absorption bands between $2.3 \mu \mathrm{m}$ and $2.4 \mu \mathrm{m}$. The HeI emission lines at $2.06 \mu \mathrm{m}$ and $2.11 \mu \mathrm{m}$, some of them showing P-Cygni profiles and/or broad line wings, represent the class of luminous massive stars during their short post main sequence wind phase. About 18 of these stars have been found in the Galactic center, some of them even exhibiting HeII $(2.189 \mu \mathrm{m})$ emission (Allen et al. 1990; Burton \& Allen 1992; Krabbe et al. 1991, 1995b; Blum et al. 1995; Libonate et al. 1995; Tamblyn et al. 1996). Wolf-Rayet stars of subtypes WN9/WC9 or Ofpe are identified by their HeI emission lines together with NIII (2.11 $\mu \mathrm{m})$ or CIII $(2.11 \mu \mathrm{m}, 2.324 \mu \mathrm{m})$.

25 stars from the central field were classified and placed on a HR-diagram. A stellar population synthesis model (Krabbe et al. 1994) modified for the higher metallicity in the Galactic Center was fitted to the distribution. Based on these data, the most likely scenario for the formation of the massive stars is a small starburst between 4 and $810^{6}$ years ago. Figure 10 displays two very red spectra. These recently formed, therefore still highly obscured, massive stars may have been transported into the central core along with orbiting gas streamers.

In addition to classifying the stars, we cross-correlated the spectra and determined the velocity dispersion of the cluster stars. Including earlier data of some stars further out (Rieke \& Rieke 1988; Sellgren et al. 1987), the radial velocity dispersion of 35 early and late type stars with distances of $1^{\prime \prime}$ to $12^{\prime \prime}$ from the dynamical center ( $\mathrm{SgrA}^{*}$ ) is $159 \pm 19 \mathrm{~km} / \mathrm{s}$. This result strongly favours the existence of a central dark mass of $\sim 310^{6} M_{\odot}$. within $\sim 0.14 \mathrm{pc}$ of the dynamic center (Krabbe et al. 1995b; Haller et al. 1996).

\subsection{IC 342}

IC 342 is a nearby classical starburst galaxy and is well suited for a detailed investigation due to its small distance of about 1.8 Mpc, its almost face-on orientation and its much lower internal extinction compared to, for example, M82. In its central region, IC 342 shows many typical signs of ongoing star bursts which made it a target for many investigations, especially in the sub-mm and radio regime. IC 342 shows a molecular bar in ${ }^{12} \mathrm{CO}$ (Lo et al. 1984; Eckart et al. 1990; Wright et al. 1993), ${ }^{13} \mathrm{CO}$ (Turner \& Hurt 1992), and HCN (Downes et al. 1992), which peaks 
Fig. 10. Galactic Center: $K$-band spectra of central cluster stars. From the spectral type of many stars the history of the cluster can be determined by fitting its properties with stellar population synthesis starburst models. The mass of central dark matter can be assessed from the radial function of the velocity dispersion of the cluster members (Krabbe et al. 1995b; Genzel et al. 1996). (To be seen in landscape) 


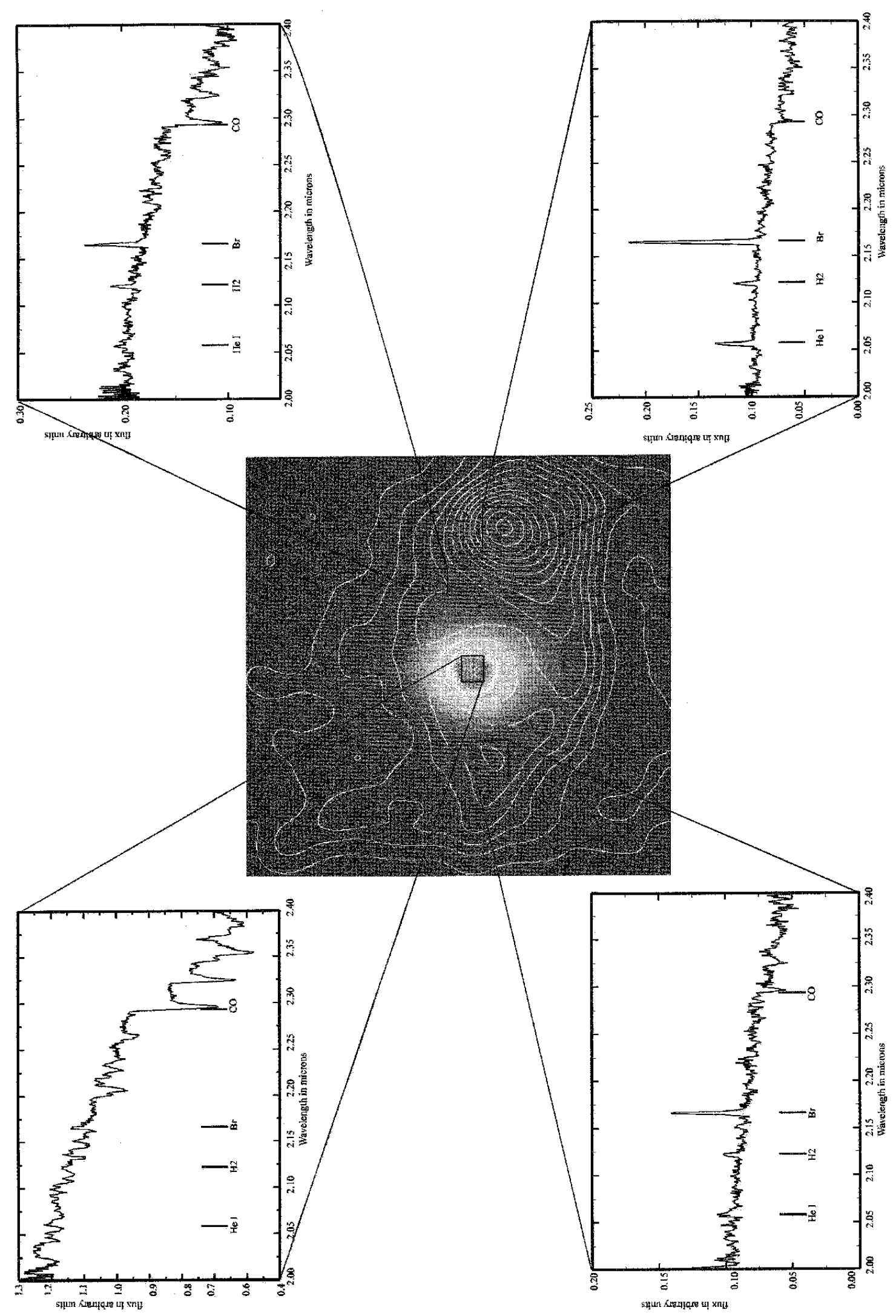

Fig. 11. Spectra of various regions in the central $100 \mathrm{pc}$ of IC 342 . The grey scale image $\left(12^{\prime \prime} \times 12^{\prime \prime}\right)$ represents the $K$-band continuum, the $\operatorname{Br} \gamma(2.16 \mu \mathrm{m})$ line emission is overlaid in linearily spaced contours. The spectra have been extracted from the data set at the location shown on the image and averaged over the boxes. The spectral lines clearly reveal strong spatial variations of the stellar population and the star burst activity across the nucleus (Böker et al. 1996). (To be seen in landscape) 
at the NIR-continuum center and extends in the northsouth direction for about $1^{\prime}$. The central region, viewed in all of these molecular lines, has a ring-like shape with a diameter of about $6^{\prime \prime}$ which may be the ends of the spiral arms interacting with the ends of the bar.

3D data have been obtained at the $3.5 \mathrm{~m}$ telescope on Calar Alto at 4 spatial positions on IC 342 covering most of the central $12^{\prime \prime}$ and revealing a wealth of information. Figure 11, displaying the $K$-band spectra at different positions toward and in the vicinity of IC 342's nucleus, shows clearly how the physical conditions vary in and along the starburst ring. A continuum subtracted channel map at the position of the Brackett $\gamma$ emission line, shown in Fig. 11 in contours, traces the morphology of the starburst ring very nicely and demonstrates how the data cube can be investigated in detail. A detailed analysis will be given in Böker (1996).

\subsection{IRAS F10214+4724}

At a redshift of 2.284 the galaxy IRAS F10214+4724 (hereafter F10214) is suggested to be one of the most luminous single objects in the universe. A bolometric luminosity of $L_{\mathrm{bol}}>310^{14} L_{\odot}\left(H_{0}=50\right.$, Rowan-Robinson et al. 1991) and a large mass of molecular gas $\left(\sim 210^{11} M_{\odot}\right.$, $H_{0}=100$; Solomon et al. 1992) has led to speculation that this source represents a true primeval galaxy in the process of forming the bulk of its stellar population and enriching its ISM with metals for the first time. However, the distorted arc-like appearance of F10214 in high resolution $K$-band images from the Keck telescope (Matthews et al. 1994; Graham \& Liu 1995) and in recent optical HST imaging (Eisenhardt et al. 1995), the presence of companions, and statistical arguments makes it almost certain that F10214 is gravitationally lensed by a foreground object (Broadhurst \& Lehár 1995; Eisenhardt et al. 1995). Both UV as well as optical rest frame long slit spectroscopy of Elston et al. (1994) shows characteristics of an AGN-type object. Thus the nature and evolutionary state of this galaxy remains unclear.

Figure 12 shows a $K$-band spectrum (Kroker et al. 1996a), which results from $10000 \mathrm{~s}$ of integration, obtained at the $3.5 \mathrm{~m}$-telescope on Calar Alto (Spain). The $3 \mathrm{D}$ sensitivity quoted at the end of the technical section are partly based on this data set. The spectrum has the highest spectral resolution so far (compare to Elston et al. 1994) and not only clearly separates $\mathrm{H} \alpha$ and one [NII] line but shows indications of the [SII] and [OI] lines as well. The continuum map (sum of the continuum channels only) shows two other accompanying galaxies which also appear in the $K_{\mathrm{s}}$-band map of Matthews et al. (1994) and Graham \& Liu (1995). Our data do not reveal any strong $K$-band emission or absorption lines from these galaxies and their spectral energy distribution is consistent with those of early type elliptical galaxies at a redshift of $z=0.9$ (Eisenhardt et al. 1995).
This suggests that they might be foreground galaxies at a much lower redshift.

Line emission only seems to be emitted from the region of the arc. From Fig. 12 and the channel maps (not shown) which spatially resolve the arc structure, we find different morphologies of $\mathrm{H} \alpha$ and [NII] (Kroker et al. 1996a): $\mathrm{H} \alpha$ shows a compact broad component and, in addition, narrow line emission which is significantly extended in the east-west direction, following the arc in the high resolution $K$-band maps (e.g. Graham \& Liu 1995). The [NII] line emission is more compact with only very faint extensions. The ratio of the compact [NII]/broad $\mathrm{H} \alpha$ is 1.7, which is typical for AGN, where-as the ratio of the faint extended [NII]/narrow $\mathrm{H} \alpha$ is 0.5 , more typical for star forming regions. From model calculations, Broadhurst \& Lehár (1995) estimated gravitational lensing magnification factors of 50 for the nucleus and 5 for the extended regions of the host galaxy. Taking these corrections into account, we conclude that the intrinsic luminosity of F10214 is dominated by the radiation of a starburst region approximately $1-2 \mathrm{kpc}$ in diameter. This starburst produces the extended $\mathrm{H} \alpha$ emission and the far-IR flux, consistent with the detection of extended CO-emission by Downes et al. (1995). The AGN component only contributes about a third of the total flux within the $K$-band. F10214 may then be a lensed composite object with an extended starburst region and an AGN-type nucleus.

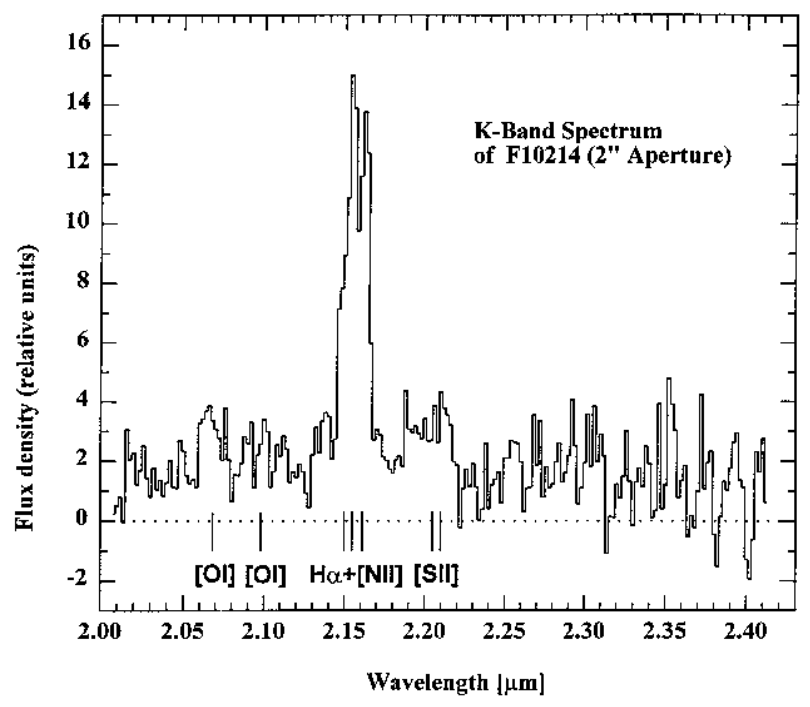

Fig. 12. $K$-band spectrum of F10214+4724 at a redshift of 2.284 (Kroker et al. 1996). The noise in the spectrum rises from $2.1 \mu \mathrm{m}$ to $2.4 \mu \mathrm{m}$ by a factor of more than 2 due to warm $300 \mathrm{~K}$ telescope background radiation. $\mathrm{H} \alpha$ is clearly separated from one of the $[\mathrm{NII}]$ lines. $[\mathrm{OI}]$ and the $[\mathrm{SII}]$ lines are marginally detected

Acknowledgements. It is a pleasure to acknowledge the MPE personnel and W. Heigel's enthusiasm in the high-precision machining of the many components. We are grateful to S. 
Drapatz for many stimulating discussions and to T. Böker, who provided IC 342 data prior to publication. We thank the staff at the 3.5-m telescope on Calar Alto, at the William Herschel Telescope on La Palma, and at the 2.2-m ESO telescope for their generous support of the observations.

\section{References}

Allen D.A., 1994, in Infrared Astronomy with Arrays: The Next Generation. In: McLean I.S. (ed.). Kluver Academic, Dordrecht, p. 49

Allen D.A., Hyland A.R., Hillier D.J., 1990, MNRAS 244, 707

Arribas S., Mediavilla E., Rasilla J.L., 1991, ApJ 369, 260

Barden S.C., Wade R.A., 1988, in Fiber Optics in Astronomy, ASP Conf. Ser. 3. In: Barden S.C. (ed.)

Blietz M., Cameron M., Drapatz S., Genzel R., Krabbe A., van der Werf P., Sternberg A., Ward M.J., 1994, ApJ Lett. 421, 92

Blum R.D., DePoy D.L., Sellgren K., 1995, ApJ 441, 603

Böker T., 1996, Ph. D. Thesis, Ludwig-Maximilian-Universität München

Broadhurst T., Lehár J., 1995, ApJ 450, L41

Burton M.G., Allen D.A., 1992, Proc. Astr. Soc. Aust. 10, 55

Dallier R., Cuby J.-G., Czarny J., Baudrand J., 1994, in: Infrared Astronomy with Arrays: The Next Generation. In: McLean I. (ed.). Dordrecht: Kluwer, p. 343

Decker H., D'Odorico S., Arsenault R., 1988, A\&A 189, 353

Downes D., Radford S.J.E., Guilloteau S., Guelin M., Greve A., Morris D., 1992, A\&A 262, 424

Downes D., Solomon P.M., Radford S.J.E, 1995, ApJ 453, 65

Eckart A., Downes D., Genzel R., Harris A.I., Jaffe D.T., Wild W., 1990, ApJ 348, 434

Eckart A., Genzel R., Hofmann R., Sams B.J., Tacconi-Garman L.E., 1995, ApJ 445, L23

Eisenhardt P.R., Armus L., Hogg D.W., Soifer B.T., Neugebauer G., Werner M.W., 1996, ApJ 461, 72

Elston R., McCarthy P.J., Eisenhardt P., et al., 1994, AJ 107, 910

Fowler A.M., Gatley I., 1990, ApJ Lett. 353, L33

Gatley I., Merrill M., 1994, in Infrared Astronomy with Arrays: The Next Generation. In: McLean I.S. (ed.). Kluver Academic, Dordrecht, p. 551

Geis N., 1990, Ph. D. Thesis, Ludwig-Maximilian-Universität München

Genzel R., Hollenbach D.J., Townes C.H., 1994, Rep. Prog. Phys. 57, 417

Genzel R., Thatte N., Krabbe A., Eckart A., Kroker H., Tacconi-Garman L.E., 1996, ApJ (in press)

Graham J.R., Liu M.C., 1995, ApJ 449, L29

Haller J.W., Rieke M.J., Rieke G.H., et al., 1996, ApJ 456, 194

Hofmann R., Blietz M., Duhoux Ph., Eckart A., Krabbe A., Rotaciuc V., 1992, in Progress in Telescope and Instrumentation Technologies, ESO Conf. proc. In: Ulrich M.-H. (ed.). ESO, Garching, p. 617
Kleinmann S.G., Hall D.N.B., 1986, ApJS 62, 501

Krabbe A., Genzel R., Drapatz S., Rotaciuc V., 1991, ApJ Lett. 382, L19

Krabbe A., Rotaciuc V., Storey J.W.V., et al., 1993, PASP 105, 1472

Krabbe A., Sternberg A., Genzel R., 1994, ApJ 425, 72

Krabbe A., Weitzel L., Kroker H., et al., 1995a, 3D - A new generation imaging spectrometer, in: Proc. of SPIE conference on: Infrared Imaging systems, April 95, Vol. 2457, Orlando, p. 172-183

Krabbe A., Genzel R., Eckart A., et al., 1995b, ApJ 447, L95

Kroker H., et al., 1996a, ApJ 463, L55

Kroker H., 1996b, Ph. D. Thesis, Ludwig-MaximilianUniversität München

Libonate S., Pipher J.L., Forrest W.J., Ashby M.L.N., 1995, ApJ 439, 202

Lord S., 1992, NASA Technical Memorandum 103957, Ames Research Center, Moffett Field, CA

Lo K.Y., Berge G.L., Claussen M.J., et al., 1984, ApJ 282, L59

Lutz D., Krabbe A., Genzel R., 1993, ApJ 418, 244

Neviere M., 1991, Appl. Opt. 30, 4540

Neviere M., 1994, (private communication)

Matthews K., et al., 1994, ApJ Lett. 420, L13

Richardson E.H., 1972, in Auxiliary Instrumentation For Large Telescopes, ESO/CERN Conf. proc. In: Lausten S. \& Reiz A. (eds.)

Richardson E.H., Fletcher J.M., Grundmann W.A., 1972, in Very Large Telescopes, their Instrumentations and Programs, Proc. of the IAU Colloquium No. 79

Rieke G.H., Rieke M.J., 1988, ApJ 330, L33

Rowan-Robinson M., et al., 1991, Nat 351, 719

Schroeder D.J., 1987, Astron. Opt., Academic press, p. 299

Sellgren K., Hall D.N.B., Kleinmann S.G., Scoville N.Z., 1987, ApJ 317, 881

Solomon P.M., Radford S.J.E., Downes D., 1992, ApJ Lett. 398, L29

Tamblyn P., Rieke G.H., Hanson M.M., et al., 1996, ApJ 456, 206

Thatte N., Kroker H., Weitzel L., et al., 1995, in: Proc. of SPIE Conf. on: Infrared Imaging systems, April 95, Vol. 2457, Orlando, p. 228

Turner J.L., Hurt R.L., 1992, ApJ 384, 72

van der Hulst J.M., Terlouw J.P., Begeman K., Zwitser W., Roelfsema P.R., 1992, The Groningen Image Processing System, GIPSY, in: Astronomical Data Analysis Software and Systems I. In: Worall D.M., Biemesderfer C. \& Barnes J. (eds.), ASP Conf. Ser. 25, p. 131

Vanderriest C., Lemonnier J.P., 1987, in Instrumentation for Ground-Based Opt. Astron. 1987, 9th Santa Cruz Summer Workshop. In: Robinson L.B. (ed.)

Weitzel L., 1994, Ph. D. Thesis, Ludwig-MaximilianUniversität München

Wright M.C.H., Ishizuki S., Turner J.L., Ho P.T.P, Lo K.Y., 1993, ApJ 406, 470 